Two-photon Raman processes in spectral ultrabroadening of intense ultrashort light pulses

This article has been downloaded from IOPscience. Please scroll down to see the full text article.

1993 Quantum Electron. 23497

(http://iopscience.iop.org/1063-7818/23/6/A10)

The Table of Contents and more related content is available

Download details:

IP Address: 217.21.43.222

The article was downloaded on $30 / 10 / 2008$ at $14: 43$

Please note that terms and conditions apply. 


\title{
Two-photon Raman processes in spectral ultrabroadening of intense ultrashort light pulses
}

\author{
É. M. Belenov and I. P. Prokopovich
}

P. N. Lebedev Physics Institute, Russian Academy of Sciences, Moscow

(Submitted January 12, 1993)

Kvantovaya Elektron. (Moscow) 20, 577-580 (June 1993)

The spectral evolution of an intense electromagnetic pulse in the course of a coherent twophoton Raman interaction with a medium is analyzed under conditions such that the approximation of slow envelopes is not valid for the field or polarization of the medium. There exist threshold power levels at which a pronounced self-broadening of the field spectrum occurs if the field spectrum includes the frequencies $\Omega$, where $\hbar \Omega=E_{2}-E_{1}$ is the energy difference between the levels involved in the stimulated Raman scattering. The results found here agree with experimental data on the generation of a supercontinuum in various media. In particular, there is agreement in terms of the spectral self-transformation of optical solitons in optical fibers.

Since the discovery of the generation of a spectral supercontinuum ${ }^{1,2}$ and the subsequent demonstration of this effect in various media, including solids, ${ }^{1,2}$ liquids, ${ }^{1,3,4}$ and gases, ${ }^{5}$ the research on this effect has fallen far short of giving us a complete understanding. ${ }^{5-9}$ All the theoretical models which have been developed have run into serious difficulties. ${ }^{5,9}$ None of them is versatile enough to explain the existence of common characteristics in such diverse media. The observation of a supercontinuum in a medium with a quadratic nonlinearity in the absence of self-focusing ${ }^{10}$ indicates that the self-focusing which accompanies this effect in centrally symmetric media ${ }^{1-5,7}$ serves only to reach the threshold power levels required for the development of certain processes in picosecond pulses which lead to the formation of a supercontinuum. Furthermore, experiments on the generation of a supercontinuum by femtosecond pulses over short interaction distances have shown that the spectrum of this supercontinuum does not depend on the emission angle. The supercontinuum itself retains the direction and divergence of the pump beam. ${ }^{11}$ In other words, the self-focusing and the propagation effects play nothing approaching a major role in the generation of the supercontinuum.

It was mentioned in Ref. 4 that many properties of this supercontinuum cannot be explained unless stimulated Raman scattering is taken into account. Although the supercontinuum was subsequently observed in the inert gases ${ }^{5}$ $\mathrm{Ar}, \mathrm{Kr}$, and $\mathrm{Xe}$, stimulated-Raman processes can also occur in them, since these atoms have an interaction potential well by virtue of the van der Waals force. The depth of this well reaches several tenths of $1 \mathrm{eV}$ (Ref. 12), as can be seen in gases at pressures of tens of atmospheres, which are characteristic of the experiments described in Ref. 5. Furthermore, in the fields $\sim 10^{13} \mathrm{~W} / \mathrm{cm}^{2}$ and above which which were used in Ref. 5, the energy levels of an atom, in particular, their splitting, are substantially deformed. As in the case of ordinary stimulated Raman scattering, there can be a coherent Raman scattering. ${ }^{13-15}$ Interestingly, the model of the dynamic processes which was proposed in Ref. 16 to explain the results of Ref. 5 leads to results which are quite at odds with the experimental data, as was emphasized in Refs. 15 and 16.

In this paper we start from the assumption that a selfbroadening of the field spectrum occurs in a medium in which, for some reason or other, stimulated Raman scattering occurs as intense short light pulses with a length $\tau_{p}$ satisfying $\tau_{p} \Omega<1$ propagate through the medium. This assumption is based on the results of a theoretical analysis and also a comparison of the theory proposed for the spectral self-transformation of an intense ultrashort light pulse with experiments on the formation of a supercontinuum. We might also point out that in comparatively long pulses (in the picosecond range) a supercontinuum has been observed when certain power thresholds were reached; in some cases, the effect has resulted from a self-focusing of the field. As it turns out, however, soliton-like ultrashort pulses can form under these conditions. ${ }^{17-19}$ According to the model which we have proposed, such pulses also generate a supercontinuum. If the process is accompanied by Cerenkov radiation, the blue part of the spectrum at the exit from the medium will be further than the red part from the axis of the original beam (in this regard the situation differs from the case of four-wave mixing, if this process is invoked in order to explain the generation of a supercontinuum).

In deriving a theory we start with the following assumptions. A pulse with a frequency $\omega$ and an initial length $\tau_{p 0}$, whose field $\overrightarrow{\mathscr{C}}(t, z)$ is perpendicular to the propagation direction (the $z$ axis of a Cartesian coordinate system), excites a Raman-active medium which occupies the halfspace $z>0$.

The motion of the pulse is described by the wave equation 


$$
\frac{\partial^{2} \mathscr{C}}{\partial z^{2}}-\frac{1 \partial^{2} \mathscr{C}}{v^{2} \partial t^{2}}=\frac{4 \pi}{c^{2}} \frac{\partial^{2} P}{\partial t^{2}}
$$

where $v$ is the velocity in the medium for a weak field, and $P$ is the polarization of the Raman-active medium, which is a functional of the field. The constitutive equations of the medium are

$$
\begin{aligned}
& M\left(\frac{\partial^{2} Q}{\partial t^{2}}+\Omega^{2} Q\right)=-\frac{1}{2}\left(\frac{\partial \alpha}{\partial Q}\right) \mathscr{E}^{2} \rho, \\
& \frac{\partial \rho}{\partial t}=\frac{1}{\hbar \Omega}\left(\frac{\partial \alpha}{\partial Q}\right) \mathscr{E}^{2} \frac{\partial Q}{\partial t} .
\end{aligned}
$$

Written on the left side of Eq. (2) is an oscillator function corresponding to a normal mode with a coordinate $Q$, an effective mass $M$, and an eigenfrequency $\Omega=\left(E_{2}-E_{1}\right) \hbar$. By assumption we have $\Omega \tau_{p}(z)<1$, and we have omitted a relaxation term $M\left(2 / T_{2}\right)(\partial Q / \partial t)$ from (2). On the right side of (2) is a driving force which is proportional to the derivative of the polarizability $\alpha(Q)$ of the oscillator near the equilibrium value $Q=Q_{0}$ and the difference $\rho(t, z)$ between the populations of the levels $E_{2}$ and $E_{1}$, which are involved in the Raman scattering. In the absence of a field $(t<0)$ we have $\rho(t, z)=-1$; this situation corresponds to the case of an unexcited medium. When there is a field, the dynamics of $\rho(t, z)$ is described by Eq. (3). Depending on the particular physical situation, Eqs. (1)-(3) can describe different processes, e.g., a scattering of light by molecular vibrations of a gas with a polarizability $\alpha(Q)$ which depends on the distance $(Q)$ between the nuclei, a scattering by optical phonons in a solid, an interaction of a pulse with a two-level system in which a transition is forbidden in the electric-dipole approximation, Raman scattering accompanied by spin flip, Raman emission in a multicomponent plasma, scattering by coupled electron-phonon excitations, Brillouin scattering (as a particular case of Raman scattering by acoustic phonons), and Raman scattering in amorphous semiconductors, liquids, and glasses. There is accordingly justification for going back to the conclusion of Ref. 4 . The formation of a supercontinuum in various media is related in some way or another to stimulated Raman scattering.

The field-induced nonlinear polarization which appears in the wave equation is governed by the relation

$$
P=N\left(\frac{\partial \alpha}{\partial Q}\right) Q \mathscr{E},
$$

where the quantity $N$, whose dimensionality is reciprocal cubic centimeters, can be thought of as the number of particles per $1 \mathrm{~cm}^{3}$.

At the times $t=0$ the field $\mathscr{C}(t, z)$ is given at the $z=0$ boundary of the medium, and Eqs. (1)-(3) with relation (4) can be solved under the assumption that the shape of the pulse varies only slightly over distances on the order of the pulse length. In the calculations below we use the dimensionless coordinate $\beta z$, where $\beta=\mid 2 \pi N \rho(0, z)(\partial \alpha \mid$ $\partial Q)\left(\hbar \Omega / 2 M c^{2}\right) \mid$, the dimensionless field $E(t, z$ $=\mathscr{C}(t, z) / \mathscr{C}_{0}$, where $\mathscr{C}_{0}$ is a constant with the dimensionality of a field which we have selected, and the modulus of

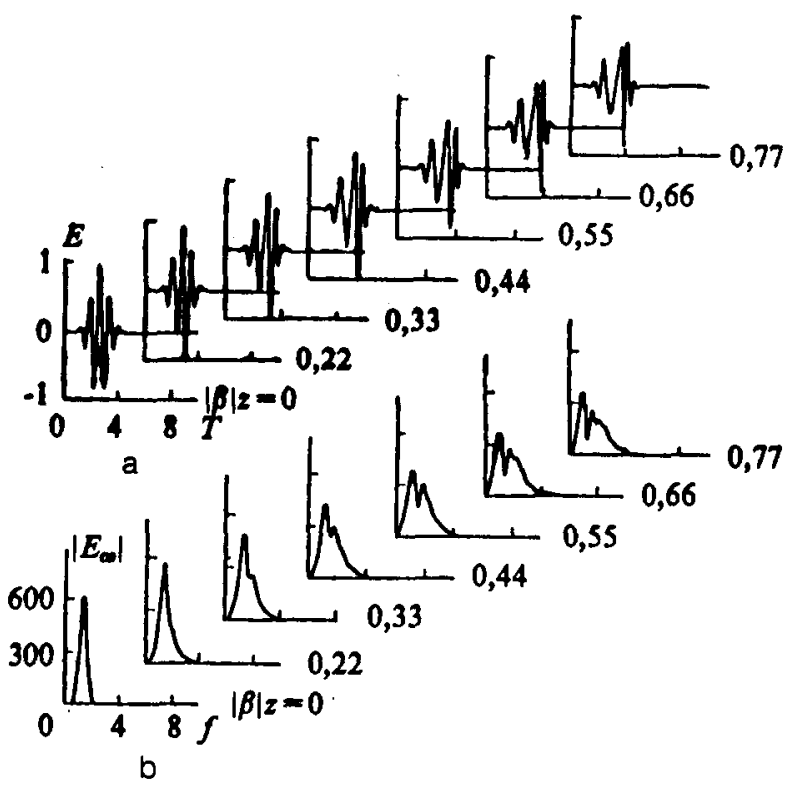

FIG. 1. Dynamics of the field $E(t, z)$ (a) and of the modulus of its spectrum, $E_{\infty}=E_{\infty}(\omega, z)(b)$, for the case $\varphi_{0}=2 \pi$ and $\Omega \tau_{\rho 0}=0.1$. The field at the entrance to the medium is given by $E(T, 0)=\sin \Omega\left(T-T_{0}\right) \exp \left\{-\left[\left(T-T_{0}\right)^{2} \log 2\right]\right\}$. The dimensionless time $T$ is expressed in units of $\tau_{\rho 0}$. The field frequency $\bar{\Omega}$ is expressed in units of $\tau_{p 0}^{-1}$. The frequency of the spectrum, $f$, is expressed in units of $T_{\infty}^{1}=\Omega / 2 \pi$. The values $\widetilde{\Omega}=9$ and $T_{0}=2.5$ are assumed.

the spectral amplitude of the field, $E_{\omega}$ (in arbitrary units). The quantity $\beta^{-1}$, with the dimensionality of a length, can be called the "Raman self-scattering length" of the pulse.

An important role is played in the theory by the phase

$$
\varphi(t, z)=\left(\frac{\partial \alpha}{\partial Q}\right)(2 \hbar \Omega M)^{-1 / 2} \int_{-\infty}^{t} \mathscr{E}^{2}\left(t^{\prime}, z\right) d t^{\prime},
$$

which can be likened to the Rabi frequency in the case of single-photon coherent interactions of a two-level system with a field. For example, for the normalization of $\varphi(t, z)$ in (5), the population difference $\rho(t, z)$ between the Raman levels varies in accordance with the following equation under the condition $\Omega \tau<1$ (see, for example, Ref. 20 ):

$$
\rho(t, z)=-\cos \varphi(t, z) .
$$

The field spectrum expands rapidly in both directions. In addition, as can be seen from Fig. 1b, the spectrum is asymmetric, in contrast with the spectrum of the input pulse. This effect again agrees with the experiments of Ref. 8 on the generation of a supercontinuum. As it evolves, the spectrum becomes a discrete-continuous spectrum with equidistant peaks; its intensity falls off more rapidly in the high-frequency region (Fig. 1b), in agreement with both the experiments of Ref. 8 and experiments on anomalous spectral broadening. ${ }^{24,25}$

Calculations show that an increase in $\Omega \tau_{p}$ under conditions of stimulated Raman scattering $\left(\Omega \tau_{p}<1\right)$ leads to an intensification of the field self-effect during the generation of the supercontinuum. 


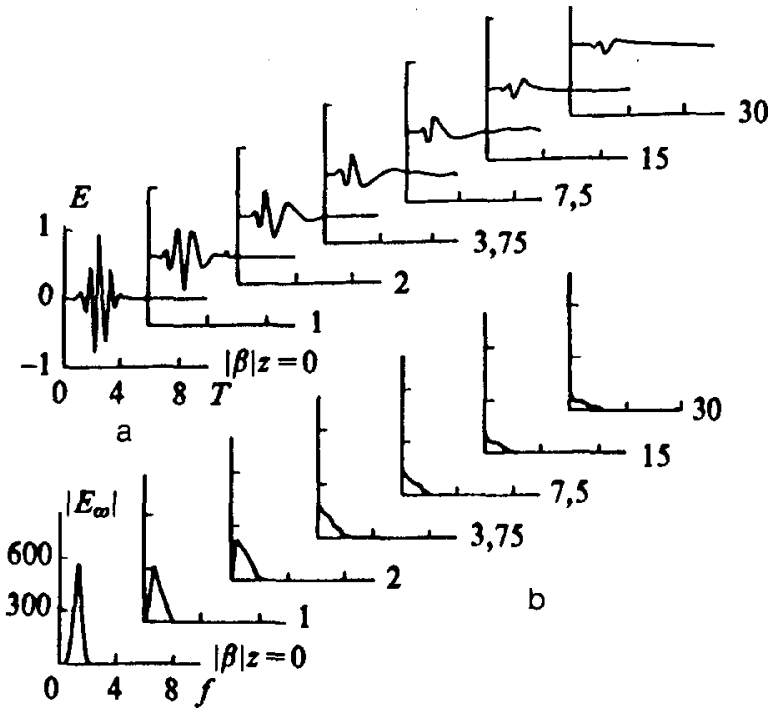

FIG. 2. Dynamics of the field $E(t, z)$ (a) and of the modulus of its spectrum, $E_{\infty}=E_{\infty}(\omega, z)(b)$, for the case $\varphi_{0}=\pi$ and $\Omega \tau_{p 0}=0.1$.

This evolution of the spectrum can be explained without difficulty by examining the case $\Omega \tau_{p} \ll 1$, in which constitutive equations (2) and (3) have the simple analytic solution in (6). Under the condition $\varphi<\pi$, there is a predominant filling of the upper level of the Raman-active medium. This case corresponds to the generation and amplification of Stokes components of the field: the center of gravity of the spectrum shifts toward lower frequencies. The condition $\varphi>\pi$, on the other hand, corresponds to the generation of anti-Stokes components [according to (6), the upper level is filled], and the spectrum of the field shifts into the blue region. If $\varphi(t, z)$ varies in the interval $0-2 \pi$, low-frequency Stokes components are generated at the leading edge of the pulse, while high-frequency anti-Stokes components are generated at the trailing edge (see the pulse shape in Fig. 1a). The spectrum thus broadens in both directions away from the original field frequency.

Analysis of Eqs. (1)-(3) and a numerical solution of these equations show that there exist phase regions $\varphi(\infty, 0)=\varphi_{0}$ which separate qualitatively different dynamics of the pulse spectrum $\left|E_{\infty}\right|$. At $\varphi_{0} \approx \pi$, for example, the pulse spectrum broadens in the low-frequency direction. Figure 2 shows the dynamics of the field $E(t, z)$ and of its spectrum $\left|E_{\omega}\right|$ for the case $\varphi_{0}=\pi$. With increasing value of the dimensionless coordinate $\beta z$, the spectrum broadens by virtue of an increase in the intensity of the red components. These results agree with experiments on the transformation of the spectrum of optical solitons in optical fibers $^{21-23}$ and experiments on the generation of a supercontinuum in comparatively low fields. In our theory, as we have already mentioned, this situation corresponds to small values of $\varphi_{0}$.

For fields with large values of $\varphi_{0}$, the spectrum broadens in both directions away from $\omega$ (Ref. 10). According to the theory, this spectral broadening corresponds to a phase $\varphi_{0}>\pi$. Figure 1 shows the evolution of the field and its spectrum with an initial phase $\varphi_{0}=2 \pi$.

For a given pulse intensity, there thus exist two threshold intensities which separate regions of different dynamics of the broadening of the pulse spectrum.

'R. R. Alfano and S. L. Shaprio, Phys. Rev. Lett. 24, 584, 592, 1217 (1970).

${ }^{2}$ N. G. Bondarenko, I. V. Eremina, and V. I. Talanov, Pis'ma Zh. Eksp. Teor. Fiz. 12, 125 (1970) [JETP Lett. 12, 85 (1970)].

${ }^{3}$ A. Penzkofer, A. Lauberau, and W. Kaiser, Phys. Rev. Lett. 31, 863 (1971).

${ }^{4}$ W. L. Smith, P. Liu, and N. Bloembergen, Rev. A 15, 2396 (1977).

${ }^{5}$ P. B. Corkum, C. Rolland, and T. Srinivasan-Rao, Phys. Rev. Lett. 57, 2268 (1986).

${ }^{6}$ G. Yang and Y. R. Shen, Opt. Lett. 9, 510 (1984).

'J. T. Manassah, M. A. Mustafa, R. R. Alfano, and P. P. Ho, IEEE J Quantum Electron. QE-22, 197 (1986).

${ }^{8}$ The Supercontinuum Laser Source (Springer-Verlag, New York, 1989).

${ }^{9}$ I. Golub, Opt. Lett. 15, 305 (1990).

${ }^{10}$ A. A. Murav'ev and A. N. Rubinov, Pis'ma Zh. Eksp. Teor. Fiz. 37, 597 (1983) [JETP Lett. 37, 713 (1983)].

${ }^{11}$ R. L. Fork, C. V. Shank, C. Hirlimann, R. Yen, and W. J. Tomlinson, Opt. Lett. 8, 1 (1983).

${ }^{12}$ L. D. Landau and E. M. Lifshitz, Quantum Mechanics [in Russian] (Nauka, Moscow, 1989) (previous editions of this book have been published in English translation by Pergamon, New York).

${ }^{13}$ M. H. Mittleman, Theory of Laser-Atom Interactions (Plenum, New York, 1982).

${ }^{14}$ Q. Su and J. H. Eberly, Phys. Rev. A 44, 5997 (1991).

${ }^{15}$ M. V. Fedorov, An Electron in an Intense Optical Field [in Russian] (Nauka, Moscow, 1991)

${ }^{16}$ M. V. Fedorov, M. Yu. Ivanov, and P. B. Lerner, J. Phys. B. 23, 2505 (1990).

${ }^{17}$ J. C. Englund and C. M. Bowden, Phys. Rev. Lett. 57, 2661 (1986); Phys. Rev. A 42, 2870 (1990).

${ }^{18}$ D. C. McPherson, R. C. Swanson, and J. L. Carlsten, Phys. Rev. A 40, 6745 (1989); R. C. Swanson, D. C. McPherson, P. R. Battle, and J. L. Carlsten, Phys. Rev. A 45, 450 (1992).

${ }^{19}$ K. Druhl, R. G. Wenzel, and J. L. Carlsten, Phys. Rev. Lett. 51, 1171 (1983).

${ }^{20}$ E. M. Belenov, A. V. Nazarkin, and I. P. Prokopovich, Pis'ma Zh. Eksp. Teor. Fiz. 55, 223 (1992) [JETP Lett. 55, 218 (1992)].

${ }^{21}$ E. M. Dianov, A. Ya. Karasik, V. P. Mamyshev et al., Pis'ma Zh Eksp. Teor. Fiz. 41, 242 (1985) [JETP Lett. 41, 294 (1985)].

${ }^{22}$ F. M. Mitchke and L: F. Mollenauer, Opt. Lett. 11, 659 (1986).

${ }^{23}$ A. B. Grudinin, E. M. Dianov, D. B. Korobkin et al., Pis'ma Zh. Eksp. Teor. Fiz. 45, 211 (1987) [JETP Lett. 45, 260 (1987)].

${ }^{24}$ F. Shimizu, Phys. Rev. Lett. 19, 1097 (1967).

${ }^{25}$ A. M. Bol'shov and G. V. Venkin, Zh. Prikl. Spektrosk. 9, 1050 (1968); M. A. Bol'shov, G. V. Venkin, S. A. Zhilkin, and I. I. Nurminskiï, Zh. Eksp. Teor. Fiz. 58, 3 (1970) [Sov. Phys. JETP 31, 1 (1970)]

Translated by $D$. Parsons 